\title{
Afferent Projections of the Trigeminal Nerve in the Goldfish, Carassius auratus
}

\author{
R.L. PUZDROWSKI \\ Department of Biology, The University of Michigan, Ann Arbor, \\ Michigan 48109
}

\begin{abstract}
The horseradish peroxidase (HRP) histochemical technique was used to examine the peripheral distribution and afferent projections of the trigeminal nerve in the goldfish, Carassius auratus. Sensory fibers of the trigeminal nerve distribute over the head via four branches. The ophthalmic branch distributes fibers to the region above the eye and naris. The maxillary and mandibular branches innervate the regions of the upper and lower lip, respectively. A fourth branch of the trigeminal nerve was demonstrated to be present in the hyomandibular trunk.

Upon entering the medulla the trigeminal afferent fibers divide into a rostromedially directed bundle and a caudally directed bundle. The rostromedially directed bundle terminates in the sensory trigeminal nucleus (STN) located within the rostral medulla. The majority of fibers turn caudally, forming the descending trigeminal tract. Fibers of the descending trigeminal tract terminate within three medullary nuclei: the nucleus of the descending trigeminal tract (NDTV), the spinal trigeminal nucleus (Spv), and the medial funicular nucleus (MFn). All projections, except for those to the MFn, are ipsilateral. Contralateral projections were observed at the level of the MFn following the labeling of the ophthalmic and maxillomandibular branches. All branches of the trigeminal nerve project to all four of the trigeminal medullary nuclei. Projections to the STN and MFn were found to be topographically organized such that the afferents of the ophthalmic branch project onto the ventral portion of these nuclei, while the afferents of the maxillo- and hyomandibular branches project to the dorsal portion of these nuclei.

Cells of the mesencephalic trigeminal nucleus were retrogradely labeled following HRP application to the ophthalmic, maxillary, and mandibular branches of the trigeminal nerve. In addition to demonstrating the ascending mesencephalic trigeminal root fibers, HRP application to the above-mentioned branches also revealed descending mesencephalic trigeminal fibers. The descending mesencephalic trigeminal fibers course caudally medial to the branchiomeric motor column and terminate in the ventromedial portion of the MFn.
\end{abstract}

As in most anamniotic gnathostomes, the trigeminal sensory nerve in teleosts consists of maxillomandibular branches, which distribute fibers to the oral region, and an ophthalmic branch, which distributes fïbers to the region dorsal to the orbit and naris. In addition, in teleosts a third branch of the trigeminal nerve, termed the ramus communicans, has been described coursing peripherally along the rostral operculum as a part of the hyomandibular trunk (Herrick, 1899; Allis, '03; Maheshwari, '65; Saxena,
'67; Harrison, '81).

In teleosts the afferent fibers of the trigeminal nerve enter the brainstem as a single root that distributes fibers to the trigeminal medullary nuclear complex (Woodburne, '36; Luiten, '75). In addition to these sensory afferents, fibers from the cells of the mesencephalic trigeminal nucleus exit the brainstem through the trigeminal root and course peripherally in the branches of the trigeminal nerve.

There have been relatively few studies uti- 
lizing modern tracing techniques to investigate the primary afferents of the sensory trigeminal nerve in teleosts. Using degeneration methods to investigate the projections of the entire trigeminal root in the carp (Cy prinus carpio), Luiten ('75) reported that the descending trigeminal tract gives off axons to four different cell groups, which he considered subdivisions of the nucleus of the descending trigeminal tract. There has been only a single study of the trigeminal sensory afferents utilizing horseradish peroxidase (HRP) techniques in two species of teleosts, Hypsoblennius and Oxylebius (von Bartheld and Meyer, '85). That study was limited to an examination of the afferent projections of the trigeminal nerve branches innervating the cirri (finger-like projections found on the dorsal surface of the head in these genera) and its findings agreed, for the most part, with the description given by Luiten ('75). The mesencephalic trigeminal nucleus has been described in a number of teleosts (Weinberg, '28; Woodburne, '36; Luiten, '75, '79). However, the peripheral distribution of the afferents of these cells has never been fully examined, nor has a mesencephalic trigeminal root ever been experimentally demonstrated in a teleost.

The purpose of the present study was to provide a complete description of the primary sensory afferents of the trigeminal nerve in a teleost, the goldfish Carassius auratus, utilizing HRP techniques. An abstract of this work has been published previously (Puzdrowski, '85).

\section{MATERIALS AND METHODS}

The results of this study are based on observations made using 32 goldfish, $C$. auratus, ranging in size from $1-12 \mathrm{~cm}$ in total length. All surgical and perfusion procedures utilized animals that were anesthetized with tricaine methane sulfonate (Sigma Chemical Co., St. Louis, MO).

Five animals were used for survey purposes. Two specimens were transcardially perfused with $0.04 \mathrm{M}$ phosphate buffer and AFA $(90 \mathrm{ml} 80 \%$ ethanol, $5 \mathrm{ml}$ stock formaldehyde, $5 \mathrm{ml}$ glacial ascetic acid); the brains were removed, embedded in Paraplast (Lancer, Distributors, St. Louis, MO), and serially sectioned in the transverse plane at $10 \mu \mathrm{m}$. These sectioned brains were subsequently stained with cresyl violet and used to examine the normal anatomy of the brainstem trigeminal centers and the mesencephalic

trigeminal nucleus. A third specimen (ap-

\section{Abbreviations}

AG

ALLN

ALLN $_{d}$

ALLN $_{\mathrm{v}}$

BS

$\mathrm{Cb}$

$\mathrm{CC}$

CL DTN

DTV

EG

FL

Fm

FR

Fs

HYO

III

II

IO

IR

IV

LLF

MA

MAN

MAX

$\mathrm{MFn}$

MLF

MR

MV

$\mathrm{nC}$

NDTV

NMLF

OpV

OT

pal

PC

PG

PN

$\mathrm{rc}_{1}$

rosl

ros,

$\mathrm{v}+\mathrm{vii}$

rot

$\mathrm{rr}$

RSVII

SG

SGN

SO

Spv

SR

STN

$T$

TL

Valv

VII

VIIm

VL

Vm

$\mathrm{Xm}$

$\mathrm{XN}$ anterior ganglion

root of the anterior lateral line nerve

dorsal root of the anterior lateral line nerve

ventral root of the anterior lateral line nerve

bulbospinal tract

cerebellum

cerebellar crest

caudal lobe of the cerebellum

dorsal tegmental nucleus

descending trigeminal tract

eminentia granularis

facial lobe

facial motor nerve

fasciculus retroflexus

facial sensory nerve

hyomandibular trunk

third ventricle

inferior lobe of the hypothalamus

infraorbital trunk

inferior reticular formation

fourth ventricle

lateral longitudinal fasciculus

Mauthner axon

mandibular branch of the infraorbital trunk

maxillary branch of the infraorbital trunk

medial funicular nucleus

medial longitudinal fasciculus

medial reticular formation

cells of the mesencephalic trigeminal

nucleus

commissural nucleus of Cajal

nucleus of the descending trigeminal tract

nucleus of the medial longitudinal

fasciculus

ventricle of the optic tectum

optic tectum

palatine ramus of the facial sensory nerve

posterior commissure

posterior cerebellar tract

preglomerular complex

periventricular nucleus of the inferior lobe

Communicating ramus of the trigeminal

nerve

lateral line ramus of the supraorbital trunk trigeminofacial ramus of the supraorbital

trunk

otic ramus of the lateral line nerve

recurrent ramus of the facial sensory nerve

root of the facial sensory nerve

secondary gustatory tract

secondary gustatory nucleus

supraorbital trunk

spinal trigeminal nucleus

superior reticular formation

sensory trigeminal nucleus

dorsal thalamus

torus longitudinalis

root of the trigeminal nerve

valvula of the cerebellum

root of the facial sensory nerve

facial motor nucleus

vagal lobe

trigeminal motor nucleus

vagal motor nucleus

vagus nerve 


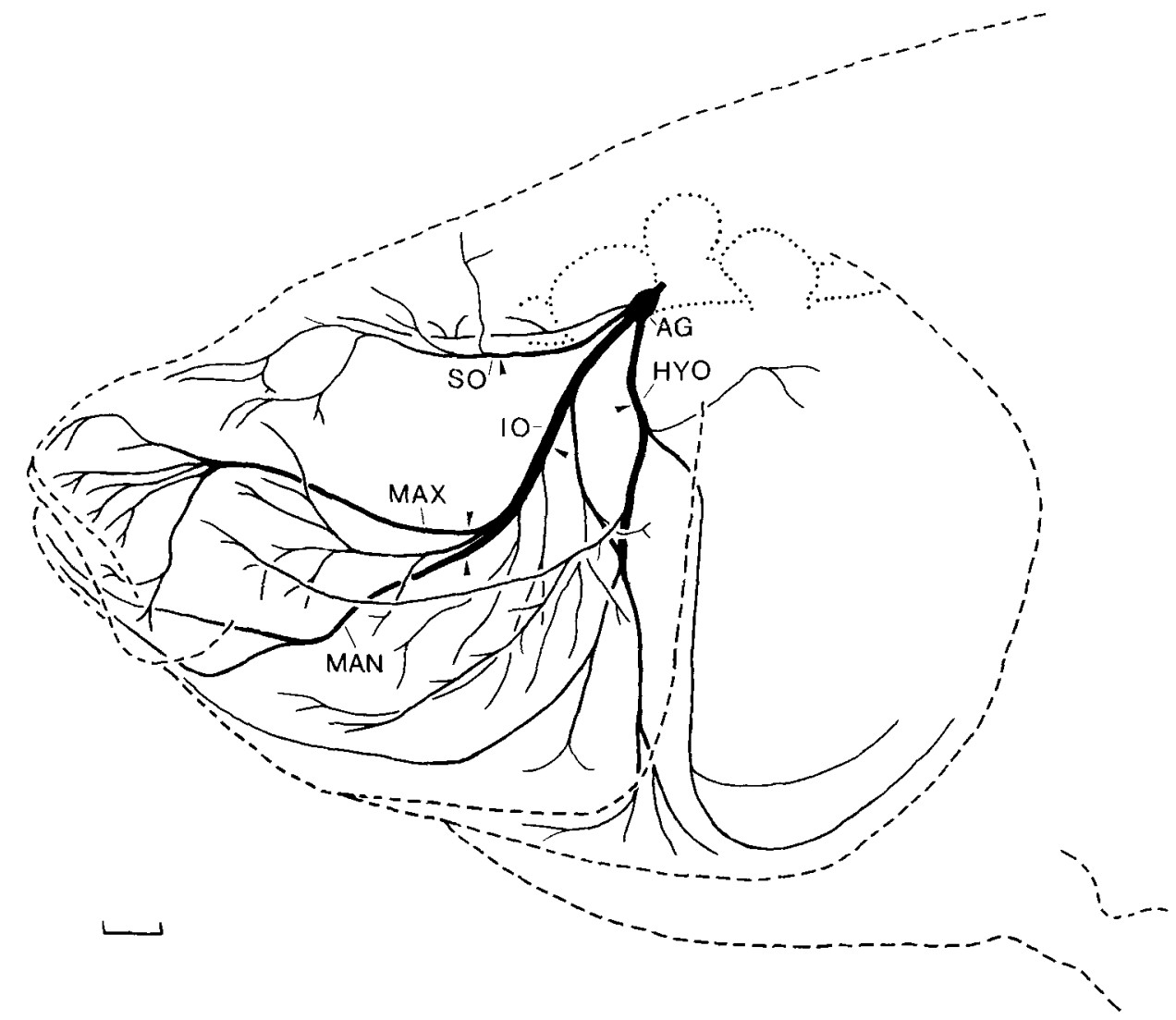

Fig. 1. Lateral view of the head of Carassius auratus illustrating the distribution of the supraorbital, infraorbital, and hyomandibular nerve trunks over the head. Arrowheads indicate points of nerve transection and

HRP application. The outline of the brain is indicated by dots; the outline of the body is indicated by dashes. Bar scale $=1 \mathrm{~mm}$

proximately $1 \mathrm{~cm}$ in length) was prepared according to the Bodian reduced silver procedure and used to examine the peripheral distribution of the main nerve trunks carrying trigeminal rami. The peripheral distribution of the nerve trunks of the head was also examined in two specimens that had been prepared by a modification of the Sihler technique (Freihofer, '66). Specimens processed by this technique are rendered transparent, except for the nerves, which are stained a dark purple.

Surgical procedures were performed with the aid of a Zeiss stereozoom operating microscope. Following anesthetization, the fish was wrapped in moistened cheesecloth and individual nerve trunks were exposed and transected. A gelfoam pledget soaked in HRP (Sigma VI, St. Louis, MO) was placed on the proximal stump of the transected nerve, and the wound was closed with histoacryl (Tri

Hawk International, Distributors, Montreal, Canada). The location of HRP application to each trunk is illustrated in Figure 1. When labeling the nerves that course along the walls of the orbit (SO, IO, MAX, and MAN, see Fig. 1) the eyeball was retracted, the nerve transected and labeled with HRP, and the eyeball then replaced and covered with histoacryl. The hyomandibular trunk was transected and labeled with HRP at the point in the rostrodorsal wall of the opercular cav. ity where it courses superficially before entering the hyomandibular bone. The palatine ramus was labeled with HRP at a point where it courses along the posterior wall of the orbit in order to establish that it was a branch of the facial sensory nerve containing no trigeminal fibers (Puzdrowski, '87).

Following a survival time of $7-14$ days at $22-26^{\circ} \mathrm{C}$, the animals were reanesthetized and transcardially perfused with cold $0.04 \mathrm{M}$ 
phosphate buffer followed by $4 \%$ glutaraldehyde. The brains were washed for $8 \mathrm{hr}$ in $20 \%$ sucrose-phosphate buffer, the meninges were removed, and the brains were embedded in gelatin. The gelatin blocks were fixed for an additional $4 \mathrm{hr}$ in $4 \%$ glutaraldehyde in $20 \%$ sucrose-phosphate buffer. The blocks were then rinsed in $20 \%$ sucrose-phosphate buffer for $8 \mathrm{hr}$ to remove any excess fixative. Forty-micrometer transverse sections of the gelatin-embedded brains were cut on a sliding microtome, collected in $0.04 \mathrm{M}$ phosphate buffer, and stored at $4^{\circ} \mathrm{C}$. The sections were processed according to the Mesulam (78) tetramethylbenzidine (TMB) protocol, or by the Hanker-Yates protocol (Hanker et al., '77) for visualization of HRP.

A total of 27 experimental cases were used in this study. Since initial unilateral experimental cases demonstrated that the trigeminal projections were for the most part ipsilateral, subsequent HRP labelings were made bilaterally. Bilateral labelings in which different nerve branches were labeled on each side of the head facilitated comparison of the relative positions of the terminal fields of individual nerves. Of the 27 experimental cases, there were 8 cases in which the supraorbital trunk (the ophthalmic branch) was labeled; 5 cases in which the infraorbital trunk was labeled; 4 cases in which the maxillary branch was labeled on one side and the mandibular branch was labeled on the other side; 5 cases in which the infraorbital trunk was labeled on one side and the supraorbital trunk was labeled on the opposite side; and 5 cases of the hyomandibular trunk, 3 in which the infraorbital trunk was labeled on the opposite side.

\section{RESULTS \\ Normal anatomy}

\section{Peripheral distribution}

The trigeminal nerve in $C$. auratus enters the brainstem as a single root comprising both sensory and motor fibers. The motor fibers compose the ventral portion and the sensory component the dorsal portion of the trigeminal root. As in many teleosts, during embryogenesis the ganglia of the trigeminal nerve fuse with the ganglia of the anterior lateral line and facial nerves to form a ganglionic complex termed the anterior ganglion. Using the nonexperimental, serially sectioned head, it was possible to distinguish the components of the ganglionic complex by tracing the cranial nerve roots of the trigeminal, facial, and anterior lateral line nerves to their respective portion of the complex (Fig. 2). The trigeminal ganglion is characterized by particularly large, pseudounipolar cells, and constitutes the extreme rostral and the rostrodorsal intermediate portion of the ganglionic plexus. Separate ophthalmic and maxillomandibular ganglia could not be distinguished.

The three cranial nerve ganglia that make up the anterior ganglion distribute rami over the head through three major nerve trunks: the supraorbital, infraorbital, and hyomandibular (Fig. 1). Application of HRP to these trunks demonstrated that each contains fibers that enter the medulla through the trigeminal root and project to the trigeminal sensory nuclei of the medulla.

The trigeminal fibers of the supraorbital trunk represent the ophthalmic branch of the trigeminal nerve. The ophthalmic branch arises from the rostral-most portion of the ganglionic complex together with a bundle of facial sensory fibers (Fig. 2). This trigeminofacial complex courses rostrally along the optic tectum and exits the braincase, along with the trochlear nerve, through a foramen lateral to the rostral pole of the tectum. After emerging from the cranium, this nerve complex is accompanied by the supraorbital branch of the anterior lateral line nerve, thus forming the supraorbital trunk. Unlike other teleosts that have been described (Herrick, 1899,1900 ), the supraorbital branch of the anterior lateral line nerve does not anastomose with the rest of the supraorbital trunk. The supraorbital trunk courses rostrally along the roof of the orbit, distributing sensory fibers to the skin of the rostrodorsal surface of the head (Fig. 1). Upon reaching the front of the orbit, the supraorbital trunk passes through a foramen in the frontal bone to distribute fibers in the region of the nasal aperture. Although branches of this trunk were observed coursing closely beneath the olfactory epithelium, no fibers were observed entering the epithelium. Furthermore, no fibers from the supraorbital trunk were traced to the oral cavity or lips.

The infraorbital trunk is composed of fibers from the trigeminal ganglion, the dorsal ganglion of the anterior lateral line nerve, and the facial ganglion. The trigeminal fibers of the infraorbital trunk comprise the maxillomandibular branches of the trigeminal nerve (Fig. 2). The infraorbital trunk courses pe- 


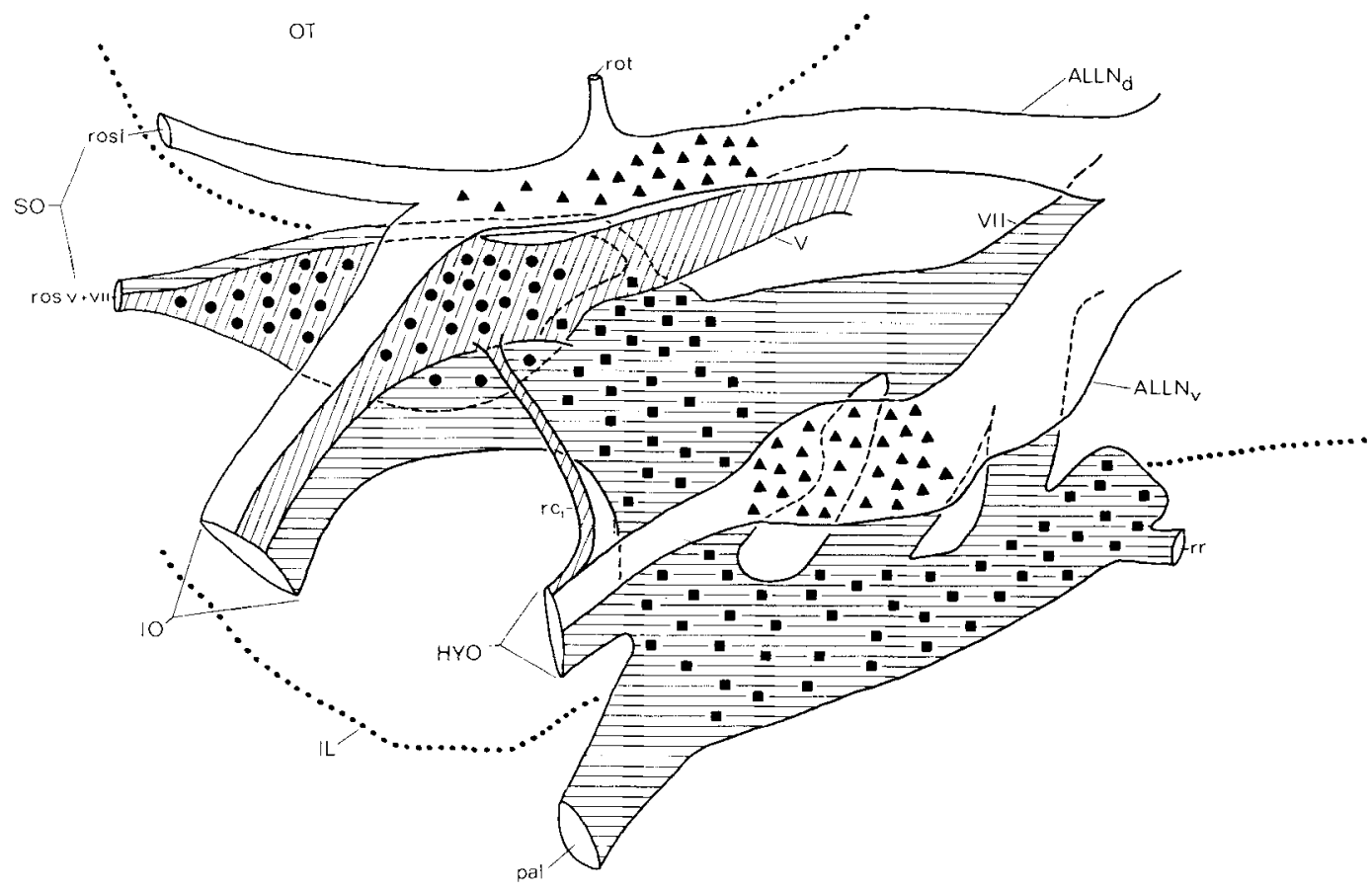

Fig. 2. Schematic drawing of the lateral view of the anterior ganglion of Carassius auratus. Rostral is to the left. To simplify the diagram only sensory components are illustrated. The relations of the trigeminal, facial, and anterior lateral line components are indicated diagrammatically. The outline of the brain is shown in small dots. The trigeminal component is represented by

ripherally along the caudal wall and floor of the orbit and divides distally into two main branches, the maxillary and mandibular (Fig. 1). Application of HRP to these branches demonstrated that each contains fibers that enter the medulla through the trigeminal root and project to trigeminal medullary centers. The maxillary branch distributes sensory fibers to the anterior roof of the mouth, the dorsal lip, and the region rostral to the eye. The mandibular branch distributes trigeminal motor fibers to the various components of the $\mathrm{m}$. adductor mandibulae, as well as sensory fibers to the floor of the mouth, lower lip, and rostral cheek region.

The trigeminal ganglion extends sensory fibers to the hyomandibular trunk by two routes. A small bundle of fibers from the trigeminal portion of the ganglionic plexus passes ventrally along the lateral edge of the trigeminal ganglion to join the hyomandibu- oblique lines; large dots indicate the position of the trigeminal ganglion. The facial component is represented by horizontal lines; squares illustrate the extent of the facial ganglion. The lateral line component is shown without highlight; triangles indicate the position of the dorsal and ventral ganglia. Bar scale $=0.3 \mathrm{~mm}$.

lar trunk ( $\mathrm{rc}_{1}$, Fig. 2). Furthermore, a small bundle of fibers separates off from the infraorbital trunk soon after this trunk exits the braincase. These fibers turn caudolaterally and join the hyomandibular trunk just before it enters the hyomandibular bone. A similar bundle of fibers, termed the ramus communicans, has been described in several other species of teleosts (Herrick, 1899; Allis, '03; Maheshwari, '65; Saxena, '67; Harrison, '81). Herrick claimed that the ramus communicans of Menidia is composed of trigeminal fibers. Whether this ramus in Carassius contains trigeminal fibers could not be determined conclusively in the present study. The peripheral distribution of the trigeminal fibers of the hyomandibular trunk was not determined in this study. However, the hyomandibular trunk distributes fibers to the opercular, gular, and cheek regions, as shown in Figure 1. 
A 4 H X.

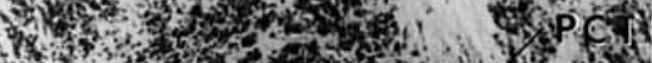

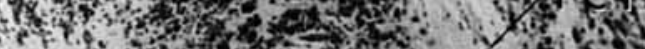
- $x^{2}=2 \mathrm{x}$

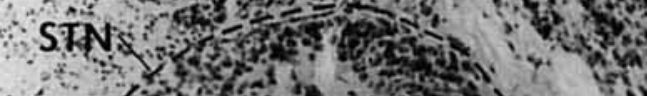

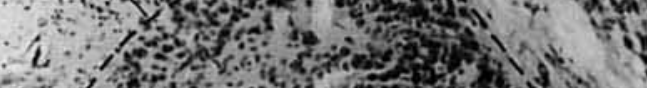
$3,1,1,0,0)$ (6) W

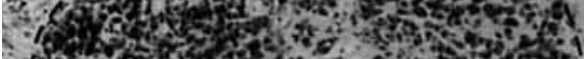
ton Why

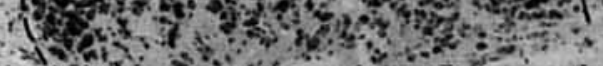

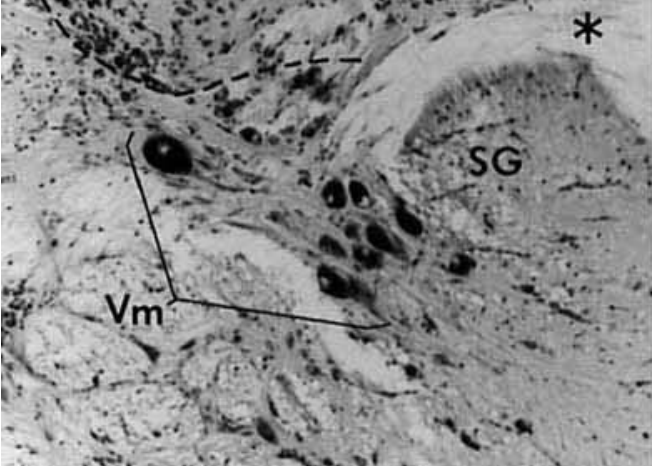

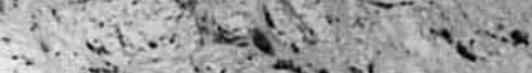

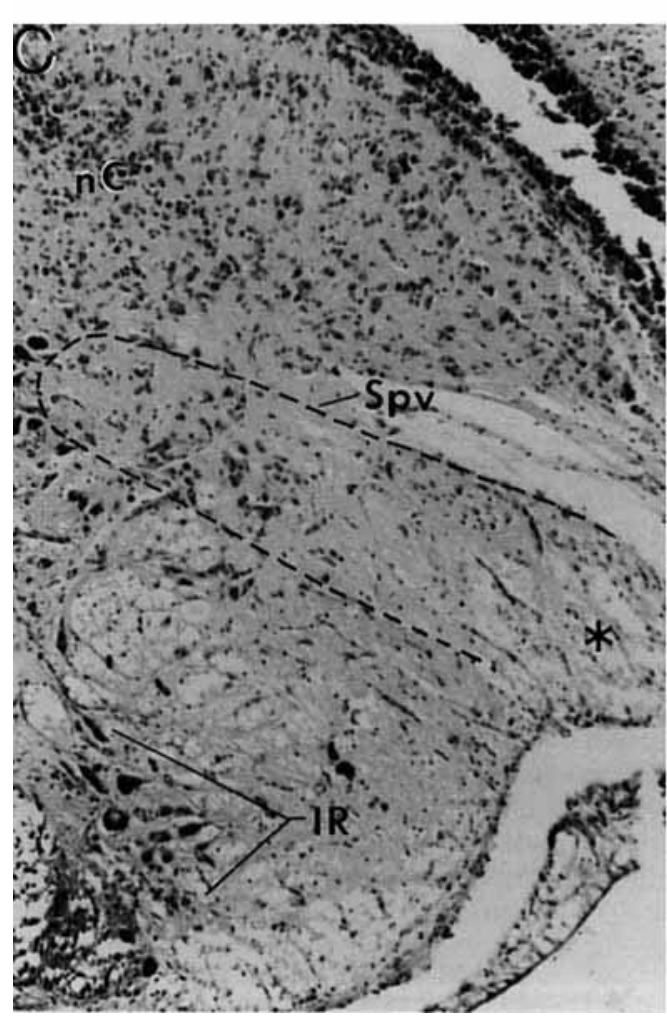

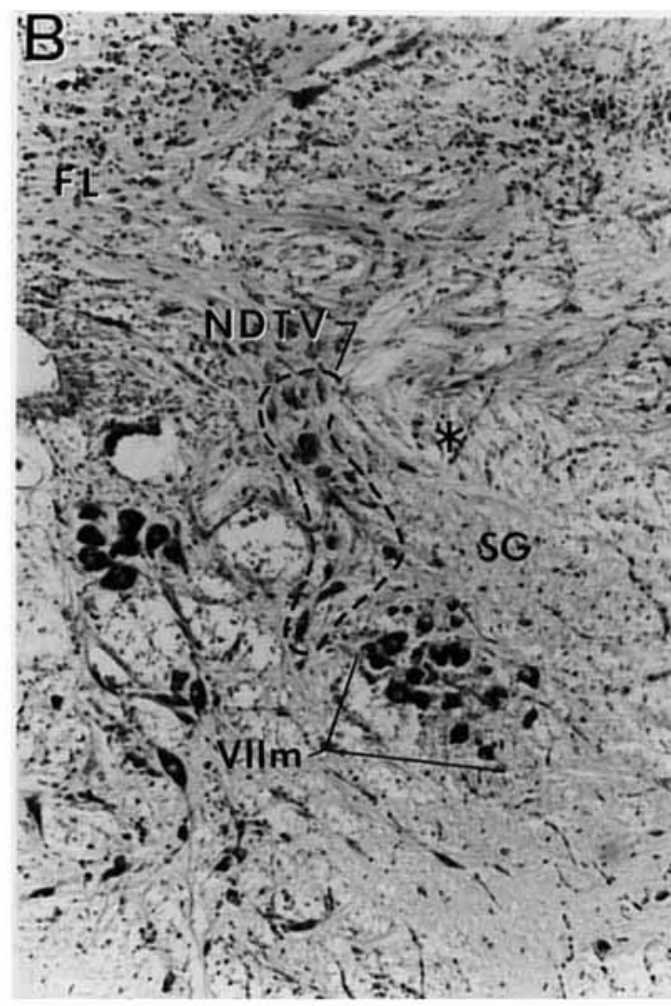

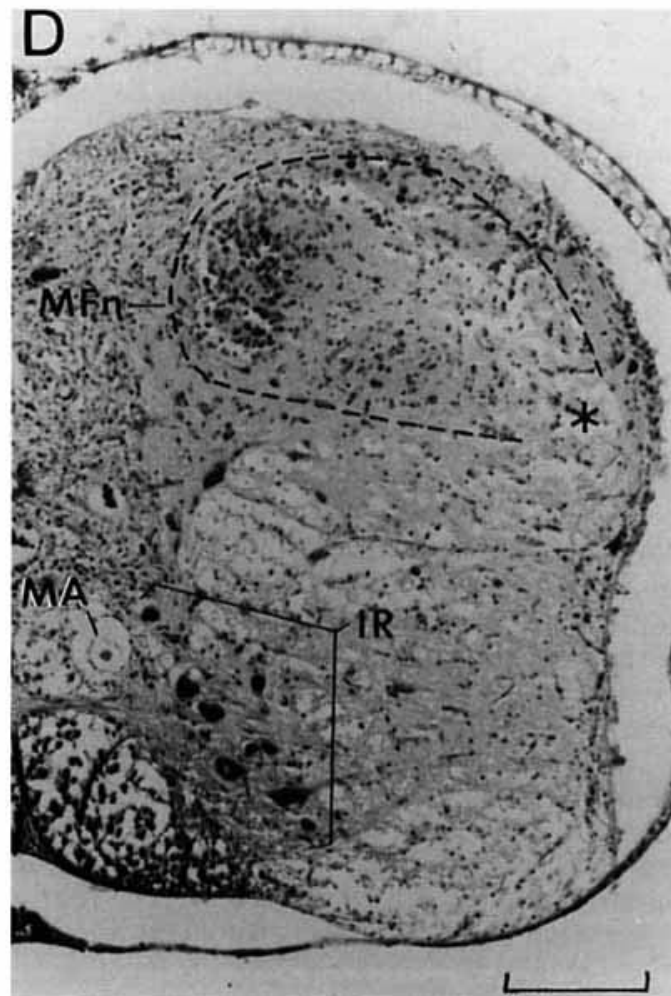




\section{The trigeminal nuclei}

The root of the trigeminal nerve enters the brainstem in a caudomedial direction and forms a cap over the ascending secondary gustatory tract (Fig. 3A). The trigeminal motor fibers continue medially and ventrally around the gustatory tract to their cells of origin in the trigeminal motor nucleus (Fig. $3 \mathrm{~A}$ ). The sensory portion of the trigeminal nerve comprises two components: the fibers to the sensory trigeminal nuclei of the medulla, and the fibers of the mesencephalic trigeminal cells. The nomenclature used for the trigeminal sensory nuclei is based on that of Herrick ('06) and Woodburne ('36). The sensory trigeminal nuclei of the medulla consist of the sensory trigeminal nucleus (STN), the nucleus of the descending trigeminal tract (NDTV), the spinal trigeminal nucleus (Spv), and the medial funicular nucleus (MFn) (Fig. 3). A more complete description of these nuclei is given with the description of the experimental results. The mesencephalic trigeminal nucleus in the goldfish is a diffuse nucleus consisting of oval-shaped cells (20$40 \mu \mathrm{m}$, long axis) which lie scattered along the floor of the ventricle of the optic tectum. The cells are found either singly or in groups of four or five in the region between the posterior commissure and the nucleus of the medial longitudinal fasciculus. The number of cells on each side of the brain varies from 8 to 12 . The mesencephalic trigeminal root was not apparent in the normal material examined in this study.

\section{Experimental results}

Since fibers from the facial and the anterior lateral nerves intermingle with the trigeminal rami to form complex nerve trunks, peripheral HRP labeling of the nerve trunks results in labeling not only the trigeminal fibers but all fibers associated with the complex. Intracranially, the fibers of each cranial nerve segregate and enter the brainstem through distinctly separate roots. It was pos-

Fig. 3. Photomicrographs of cresyl violet-stained transverse sections through the brainstem of Carassius auratus, illustrating the nuclei (outlined in dashed lines) of the trigeminal medullary nuclear complex at successive levels from A (most rostral) to D (most caudal). Medial is to the left. Asterisks indicate the position of the DTV. A: Level of the entrance of the trigeminal nerve showing the STN. B: Level of the facial motor nucleus illustrating the NDTV. C: Level of the caudal vagal root showing the Spv. D: Level of the rostral spinal cord illustrating the MFn. Bar scale in $\mathrm{D}=0.15 \mathrm{~mm}$. sible, therefore, to trace all fibers to their respective roots and thus identify the root of origin of any particular projection. Labeled fibers in the roots of the trigeminal, facial, and anterior lateral line nerves were $a b-$ served with peripheral labeling of any of the three nerve trunks. The densest trigeminal projections (i.e., the greatest number of labeled fibers) were observed following HRP application to the infraorbital trunk; the sparsest projections were observed following HRP application to the hyomandibular trunk. Each of the branches of the trigeminal nerve project ipsilaterally to all medullar nuclei (Fig. 4). Since the observed medullar projections of the maxillary and mandibular branches were similar to those following labeling of the entire infraorbital trunk, a detailed description of the infraorbital trunk projections is given and exceptions to this pattern in the maxillary and mandibular projections are noted. Ipsilateral retrograde labeling of the mesencephalic trigeminal cells was observed with HRP application to the supra- or infraorbital trunk. The efferent cells of the anterior lateral line nerves and the cells of the trigeminal and facial motor nuclei also were labeled in this study. The organization of these centers will be described in later papers.

\section{Medullar projections of the trigeminal sensory root}

The sensory trigeminal nucleus. Upon entering the brainstem a portion of the trigeminal sensory fibers passes dorsomedially and rostrally into a nucleus of densely packed, medium-sized (5-10 $\mu \mathrm{m})$, round cells (Fig. 3A), which extends from the level of the entrance of the trigeminal nerve rostrally beneath the medial portion of the secondary gustatory nucleus to the level of the commissure of the secondary gustatory nucleus. This nucleus has been termed the sensory trigeminal nucleus (STN) (Woodburne, '36). The branches of the trigeminal sensory nerve project in a rough topography onto the STN. The trigeminal sensory fibers of the ophthalmic branch project to the more ventral portion of the STN, whereas the trigeminal fibers of the infraorbital and hyomandibular trunks project to the more dorsal and rostral portion of the ST'N (Fig. 4A). The terminal fields of the infraorbital and hyomandibular branches exhibit considerable overlap within this nucleus.

The nucleus of the descending trigeminal tract. The majority of the trigeminal sensory 

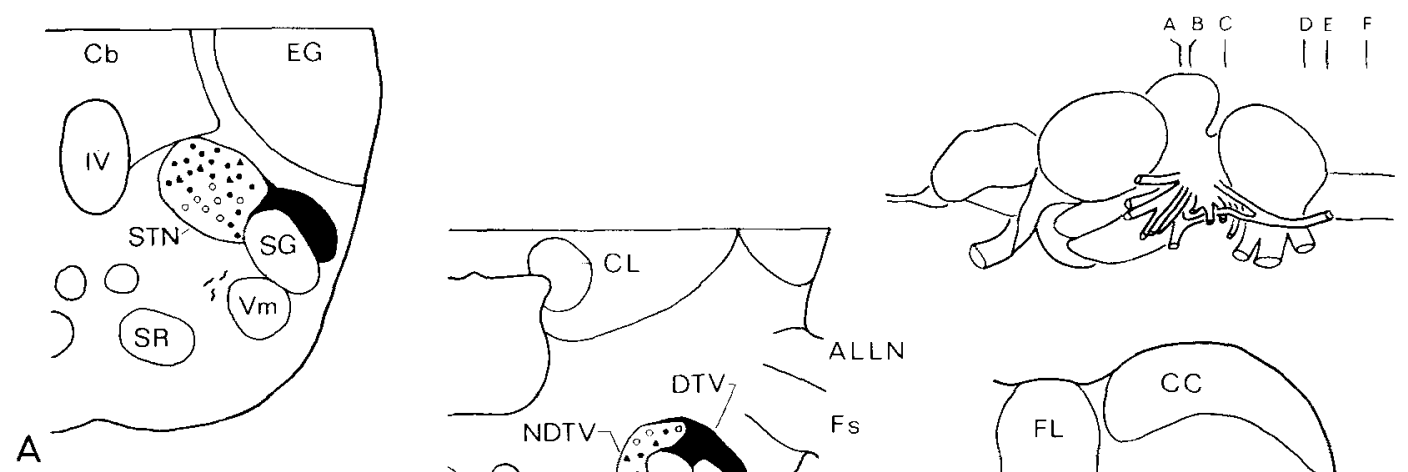

D

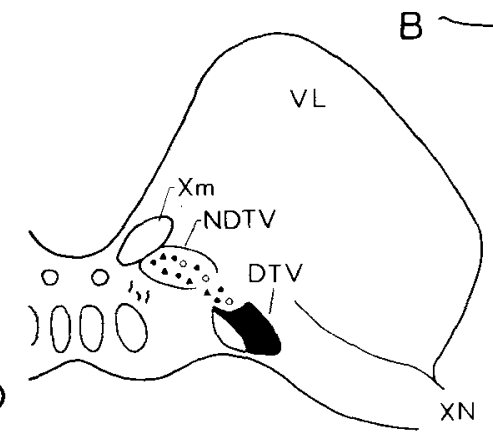

Fig. 4. Drawings of transverse sections through the brainstem of Carassius auratus illustrating the afferent projections of the ophthalmic (open circles), infraorbital (dots), and hyomandibular (triangles) branches of the trigeminal nerve. The D'TV is shown in solid black. Short

fibers turn caudally forming the descending trigeminal tract (DTV), which descends through the medulla along the dorsolateral surface of the ascending secondary gustatory tract (Fig. 4B-D). In its course caudally the DTV is divided into dorsal and ventral fascicles by the entering fibers of the facial motor root. These fascicles recombine to form a single tract immediately caudal to the facial motor root.

The DTV is accompanied by a medially situated, longitudinal column of cells termed the nucleus of the descending trigeminal tract (NDTV). The NDTV is a diffuse nucleus consisting of medium-sized $(5-10 \mu \mathrm{m})$ and large $(15-20 \mu \mathrm{m})$ round cells and large $(10$ $15 \mu \mathrm{m}$ width at midsoma) and small (5 $\mu \mathrm{m}$ wavy lines represent descending mesencephalic trigeminal fibers. Lateral view of the brain in the upper right hand indicates the level of each transverse section. Bar scale $=0.5 \mathrm{~mm}$.

width at midsoma) fusiform-shaped cells (Fig. $3 B)$. Small $(2-5 \mu \mathrm{m})$ round cells also were observed among the fibers of the descending tract. These cells have been considered to be part of the NDTV by previous investigators (Woodburne, '36; Nieuwenhuys and Pouwels, '83).

Application of HRP to any of the trigeminal nerve branches resulted in labeled fibers throughout the mediolateral extent of the DTV. As it courses caudally, the DTV sends fibers medially into the NDTV (Figs. 4, 5A,B). Whether these fibers represent collaterals of fibers within the DTV could not be determined. The projections are most extensive at four levels, which correspond to the levels of the nuclei of the branchiomeric motor col- 
umn. Each level receives projections from all trigeminal sensory branches examined. There was considerable overlap in the projections of the trigeminal nerve branches onto the NDTV. However, in general the projections of the ophthalmic branch are restricted to the dorsal portion of the NDTV, whereas those of the infraorbital and hyomandibular trunks project to both the dorsal and ventral portions of the NDTV (Figs. 4B,C, 5A,B).

Additionally, following labeling of the entire infraorbital trunk, fibers were observed coursing through the ventral portion of the NDTV to terminate among the cells of the trigeminal and facial motor nuclei (Fig. 5C). This projection was not seen following individual labeling of the maxillary or mandibular branches. A projection to the NDTV from the ventral fascicles of the facial sensory root was observed following HRP labeling of any of the three nerve trunks. This projection was heaviest following labeling of the infraorbital trunk. A projection from the DTV to the ventral aspect of the facial lobe also was seen at this level with HRP application to the infraorbital trunk (Figs, 4C, 5D). These projections are apparent with the labeling of the maxillary and mandibular branches, although in the former case they are extremely light.

The spinal trigeminal nucleus and medial funicular nucleus. The majority of fibers of the DTV descend into the caudal medulla to terminate in the spinal trigeminal nucleus (Spv) and the medial funicular nucleus (MFn). Both of these nuclei consist mostly of round cells $(5-10 \mu \mathrm{m})$ with a few small, fusiformshaped cells (Fig. 3C,D). The Spv and MFn are distinguished from the NDTV based on the lack of large, round, and fusiform-shaped cells, which are present in the NDTV. Most of the cells of the MFn are concentrated in the medial portion of the nucleus and are more densely packed than those of the Spv. The MFn extends into the rostral spinal cord to the level of the first spinal nerve.

As in the NDTV, there is a great deal of overlap between the projections of the trigeminal branches onto the Spv (Fig. 4E). In contrast, the individual branches of the trigeminal nerve project onto the $M F n$ in a distinctly topographic manner (Figs. 4F, 6). The trigeminal sensory fibers of the ophthalmic branch project to the region between the two fascicles of the infraorbital trunk (Figs. 4F, 6A). The projections of the infraorbital trunk onto the MFn are divided into a large dorsal fascicle and a smaller ventral fascicle. The ventral fascicle projects medially along the ventral border of the nucleus. The fibers of the dorsal fascicle project medially along the dorsal border of the nucleus and terminate densely in the dorsomedial portion of the MFn (Figs. 4F, 6B). Considerable overlap occurs between the ventral portion of the ophthalmic branch and the ventral fascicle of the infraorbital trunk. The trigeminal sensory fibers of the hyomandibular trunk project to the dorsolateral portion of the MFn, overlapping the dorsolateral portion of the projection area of the infraorbital trunk.

Furthermore, following HRP application to the infraorbital or ophthalmic branches of the trigeminal nerve, a small number of fibers was observed crossing the midline and coursing rostrally a short distance before terminating in the medial portion of the contralateral MFn.

The mesencephalic trigeminal root and nucleus

Labeled mesencephalic trigeminal root fibers and cells were observed following the application of HRP to the ophthalmic branch or the maxillary and mandibular branches of the infraorbital trunk (Fig. 7). The mesencephalic trigeminal root consists of large-diameter fibers that enter the brainstem with the trigeminal motor root coursing medially and ventrally toward the trigeminal motor nucleus. Although the mesencephalic trigeminal root fibers are few in number, their ascending course to the mesencephalon could be easily followed due to their large diameter (Figs. 7, 8). The mesencephalic trigeminal root does not compose a defined fiber bundle, but rather consists of scattered fibers that are spread in the mediolateral plane between the medial and lateral longitudinal fasciculi (Fig. 8). As they ascend, the mesencephalic trigeminal fibers gradually move dorsally to course beneath the dorsal tegmental nucleus toward the floor of the ventricle of the optic tectum. Furthermore, following application of HRP to the ophthalmic or infraorbital trunk branches, large-diameter fibers were observed coursing caudally, dorsolateral to the reticular formation and ventromedial to the NDTV (Figs. 4B-F, 5). In their course caudally, these fibers pass along the dorsomedial edge of the facial motor nucleus (Fig. $5 A, C, D)$. Whether or not these fibers make synaptic contacts with the cells of this motor nucleus could not be determined. The descending mesencephalic trigeminal fibers 

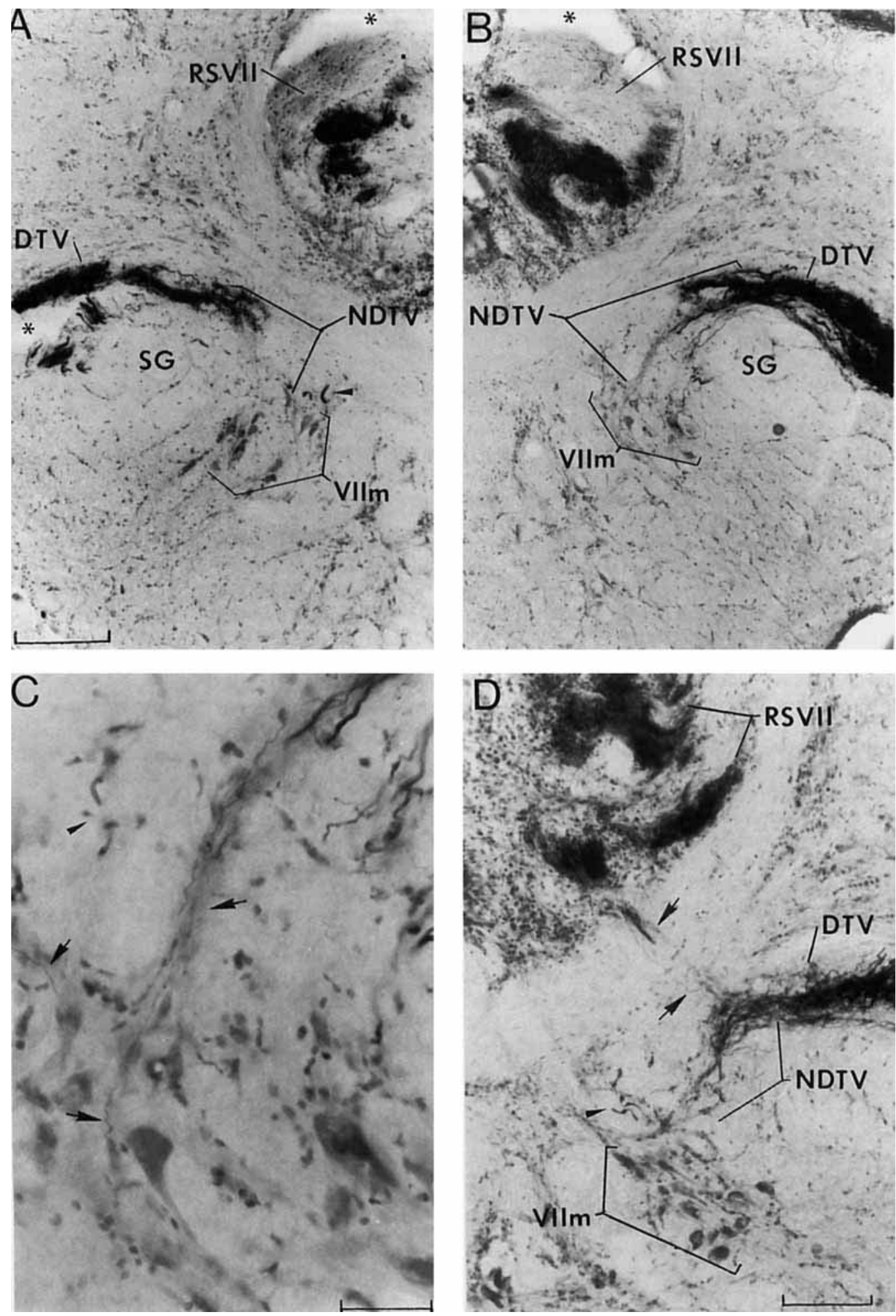
were traced into the ventromedial portion of the MFn.

There was one labeled cell in the mesencephalon for each labeled ascending mesencephalic trigeminal root fiber. The number of labeled cells following HRP application to the entire infraorbital trunk ranged from 9 to $12(\mathrm{n}=4)$. In contrast, following application of HRP to the ophthalmic branch, only 2 to 4 cells $(n=4)$ were labeled. Furthermore, individual HRP labelings of the maxillary and mandibular branches of the infraorbital trunk resulted in fewer labeled mesencephalic trigeminal cells ( 6 to $9, \mathrm{n}=4$ ) than when the entire trunk was filled more proximally. Application of HRP to the mandibular branch resulted in labeling 4 to 6 mesencephalic trigeminal cells $(n=3)$, whereas HRP application to the maxillary branch labeled only 2 to 3 cells ( $n=3$ ).

\section{DISCUSSION}

In the present investigation, the organization of the primary trigeminal sensory system of the goldfish, C. auratus, was examined. The results of this study demonstrate that the overall organization of the primary trigeminal sensory system in Carassius is similar to that in other vertebrates (Ariëns Kappers et al., '36). Furthermore, the present study serves to clarify and extend the results obtained in previous investigations of the trigeminal sensory systems of other species of teleosts (Herrick, '06; Woodburne, '36; Luiten; '75; Luiten and van der Pers, '77; von Bartheld and Meyer, '85).

In Carassius the trigeminal ganglion makes up the rostral and rostrodorsal intermediate portions of the anterior ganglionic complex. The large facial ganglion composes

Fig. 5. Photomicrographs of transverse sections through the brainstem of Carassius auratus at the level of the facial motor nucleus. A,B: Sections illustrating the projections to the NDTV 6 days after bilateral application of HRP to the ophthalmic branch (A) and infraorbital trunk (B). Small asterisks in both $A$ and $B$ indicate the shrinkage artifact. $C$ : Higher-power view of the facial motor nucleus region of the section shown in $B$, illustrating labeled fibers (large arrows) of the DTV coursing through the NDTV and among the cells of the facial motor nucleus 6 days after HRP application to the infraorbital trunk. D: Section slightly rostral to that shown in A and B, illustrating a bundle of fibers (arrows) projecting dorsomedially from the DTV toward the ventrolateral portion of the facial lobe. Small arrowheads in $\mathrm{A}, \mathrm{C}$, and $\mathrm{D}$ indicate labeled descending mesencephalic trigeminal fibers. Medial is to the left in B-D and to the right in A. Bar scale for $\mathrm{A}$ and $\mathrm{B}=0.15 \mathrm{~mm}$; for $\mathbf{C}=$ $0.04 \mathrm{~mm}$; and for $\mathrm{D}=0.10 \mathrm{~mm}$. the caudomedial portion, and the anterior lateral line ganglia lie on the dorsal and lateral edges of the anterior ganglionic complex. The organization of the anterior ganglion in the goldfish is in accordance with descriptions of the anterior ganglion of other teleosts (Herrick, 1899, 1900, '01; Luiten, '79). Further experimental investigations involving labeling of the various roots of the cranial nerves whose ganglia compose the anterior ganglionic complex are necessary to confirm these descriptions.

As in other anamniotic gnathostomes, the trigeminal sensory nerve consists peripherally of an ophthalmic branch and a maxillomandibular branch. An additional branch of the trigeminal nerve, the ramus communicans, has been reported in a variety of teleosts (Herrick, 1899, 1900, '01; Allis, 03; Maheshwari, '65; Saxena, '67; Harrison, '81). This branch has been described as coursing peripherally as part of the hyomandibular trunk. All of these reports involved nonexperimental observations of the distribution of the cranial nerves. The results of the present study confirm the presence of trigeminal sensory fibers within the hyomandibular trunk. The area of innervation of these trigeminal sensory fibers remains uncertain.

A sensory trigeminal complex, which receives primary trigeminal afferent projections, was found in Carassius extending as a continuous cell column from the isthmic region to the rostral spinal cord. This organization is in general agreement with descriptions of the trigeminal system in other teleosts (Luiten, '75; von Bartheld and Meyer, '85) and is similar to descriptions of the primary trigeminal systems in other vertebrates. In Carassius the cell column can be divided into four distinct nuclei (STN, NDTV, Spv, MFn) based on cytoarchitectural criteria. There has been some uncertainty as to whether the STN should be considered a separate nucleus or simply a rostral continuation of the NDTV. In his initial study on the trigeminal sensory system in the carp, Cyprinus carpio, Luiten ('75) considered the STN to be a subdivision of the NDTV. In a subsequent paper, Luiten and van der Pers (77) reported that the cells of the rostral portion of the STN, cells in the NDTV, SPv, and MFn project to the trigeminal and facial motor nuclei. Based on the fact that labeled cells were found only in the rostral portion of the STN it was concluded that the STN is a distinct nucleus separate from the rest of NDTV (Luiten and van der Pers, '77). In Carassius 


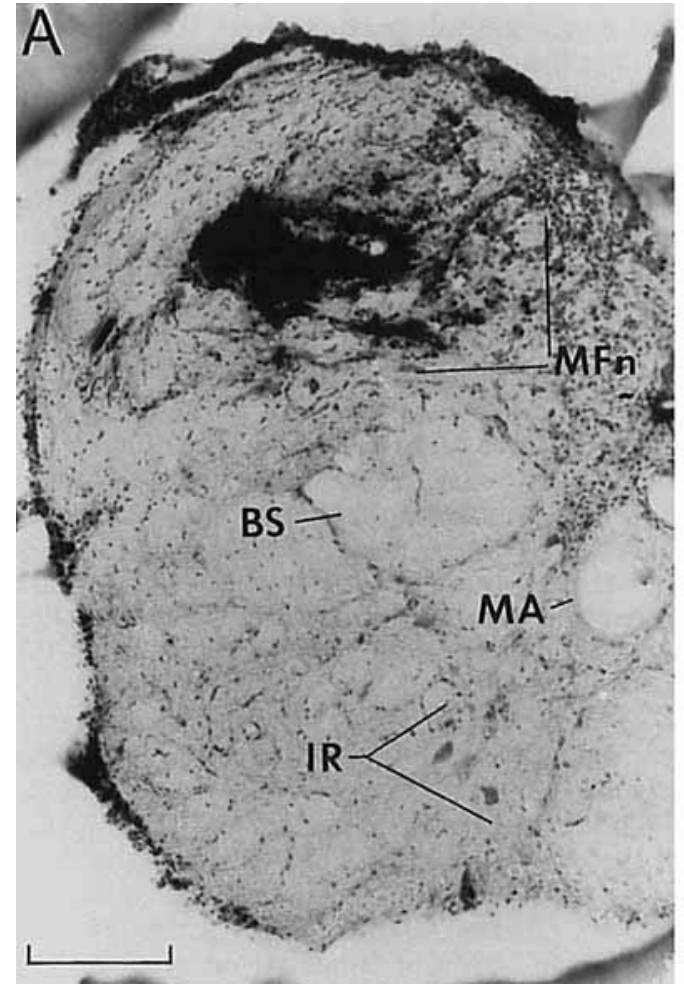

Fig. 6. Photomicrographs of the right and left half of a single transverse section through the cervical spinal cord of a goldfish illustrating the projections to the MFn 6 days after HRP application to the ophthalmic branch

the STN can be distinguished further on the basis of its cytoarchitectural organization. This nucleus is composed of densely packed, round cells, whereas in the remaining portion of the trigeminal nuclear complex the nuclei consist of relatively diffusely organized round and fusiform-shaped cells. Furthermore, in Carassius the STN has been shown to project heavily to the valvula of the cerebellum, whereas the NDTV does not (Wullimann and Northcutt, '86). These observations support the separation of the STN as a nucleus distinct from NDTV. A topologically similar primary trigeminal nucleus has been described in elasmobranchs (Smeets and Nieuwenhuys, '76; Northcutt, '78), sturgeon (New and Northcutt, '84), bullfrogs (Fuller and Ebbesson, '73), reptiles (Molenaar, '78; Barbas-Henry and Lohman, '86), birds (Dubbeldam and Karten, '78; Dubbeldam, '80), and mammals (Olszewski, '50; Kerr, '63; Marfurt, '81). Since hagfishs and lampreys appear to lack a topologically similar nucleus

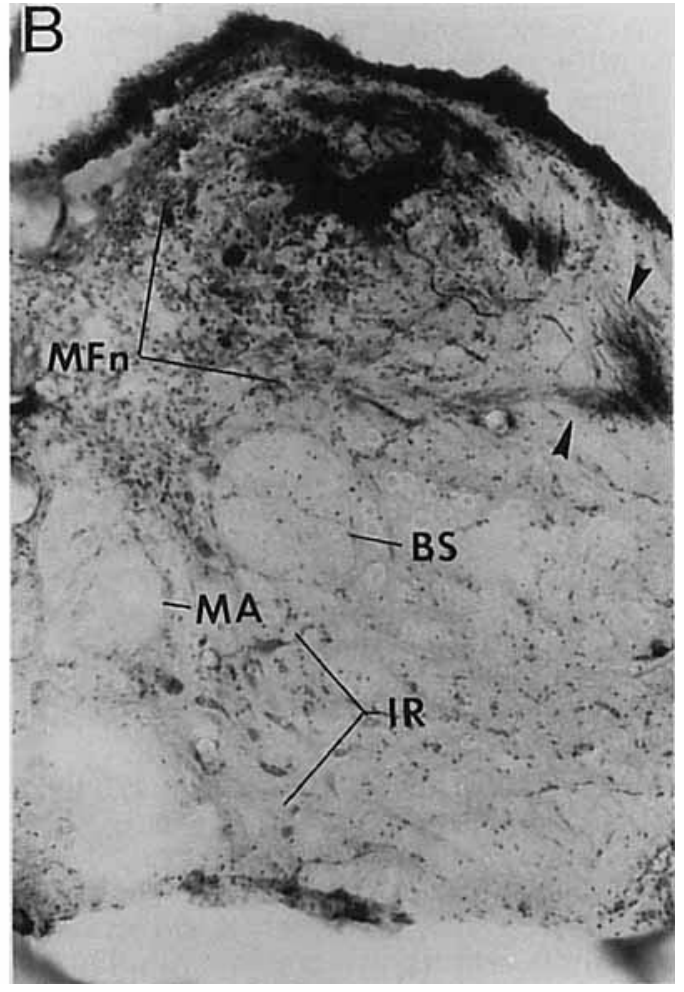

on the left side (A) and the infraorbital trunk on the right side (B) of the animal. Note the division of the projections of the infraorbital trunk into dorsal and ventral fascicles (arrowheads). Bar scale in $\mathrm{A}=0.15 \mathrm{~mm}$.

(Northcutt, '79; Ronan, '85), the STN may be a shared derived character of jawed vertebrates.

The results of the present study extend in several important ways the results obtained in previous nonexperimental and experimental studies of the trigeminal nerve afferent projections in other species of teleosts (Woodburne, '36; Luiten, '75; von Bartheld and Meyer, '85). Woodburne ('36), using nonexperimental methods to describe the trigeminal projections in trout and carp, reported a segregation of the fibers within the descending trigeminal tract such that the fibers of the ophthalmic branch course in the medial portion of the tract and the fibers of the maxillomandibular branch course in the lateral portion of the tract. In the present study no such segregation of the fibers of the DTV was observed. Labeled fibers were present throughout the mediolateral extent of the DTV following HRP application to any of the branches of the trigeminal nerve. Further- 
more, Woodburne ('36) reported a projection from the DTV to the vagal lobe. In Carassius no projection from the DTV to the vagal lobe was observed, nor have such projections been reported in other recent experimental studies of the trigeminal system in other species of teleosts (Luiten, '75; von Bartheld and Meyer, '85).

Luiten ('75), utilizing degeneration techniques, investigated the afferent projections of the entire trigeminal nerve in the carp, Cyprinus carpio. In this study Luiten reported that the DTV gives off fibers medially to five terminal fields, which he considered to be subdivisions of the NDTV. Similar results were reported by von Bartheld and Meyer ('85) in their HRP study of the central projections of the cirrus nerve (a branch of the supraorbital trunk) in Hypoblennius and
Oxylebius. The results of the present study in Carassius generally concur with these previous descriptions of the course and termination of the trigeminal afferents. Furthermore, Luiten reported a projection from the DTV to the ventrolateral aspect of the facial lobe. This projection also was found in $\mathrm{Car}$ assius with HRP labeling of the infraorbital trunk. In Carassius the ventrolateral portion of the facial lobe also receives projections from the mandibular branch of the facial sensory nerve (Puzdrowski, '87). The convergence of facial sensory and trigeminal sensory fibers on the ventrolateral portion of the facial lobe indicates that this portion of the lobe may represent an integration center for taste and touch information. Luiten and van der Pers ('77) have shown that cells in this portion of the facial lobe project to the

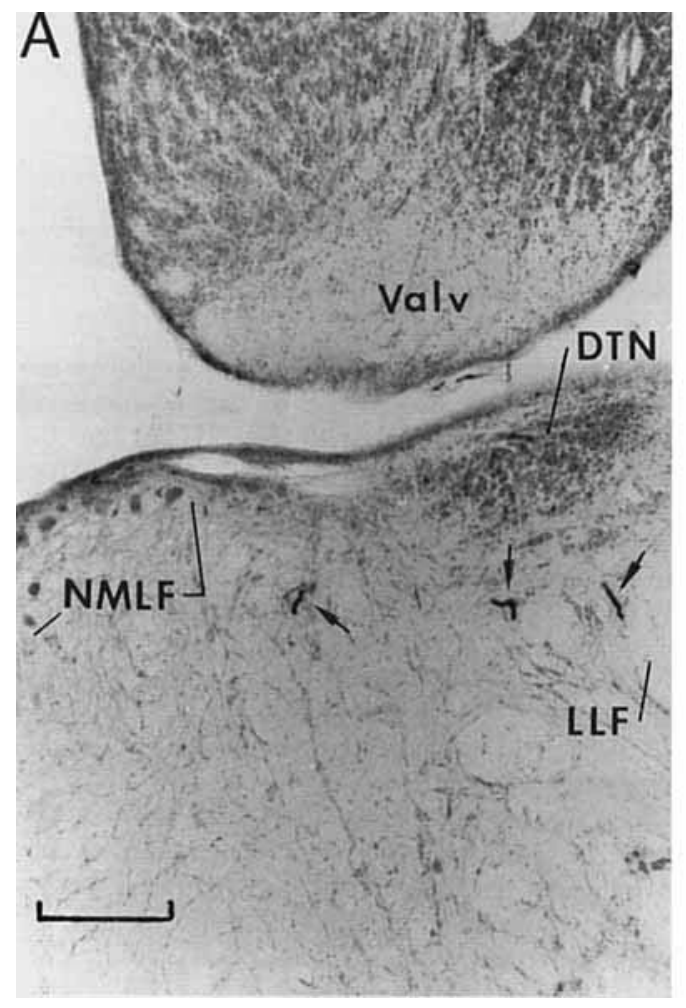

Fig. 7. Photomicrographs of transverse sections through the mesencephalon of Carassius auratus. Medial is to the left. A: Section at the level of the dorsal tegmental nucleus (DTN) illustrating labeled fibers (arrows) of the ascending mesencephalic trigeminal root coursing rostrally between the nucleus of the MLF and LLF 10 days after HRP application to the ophthalmic

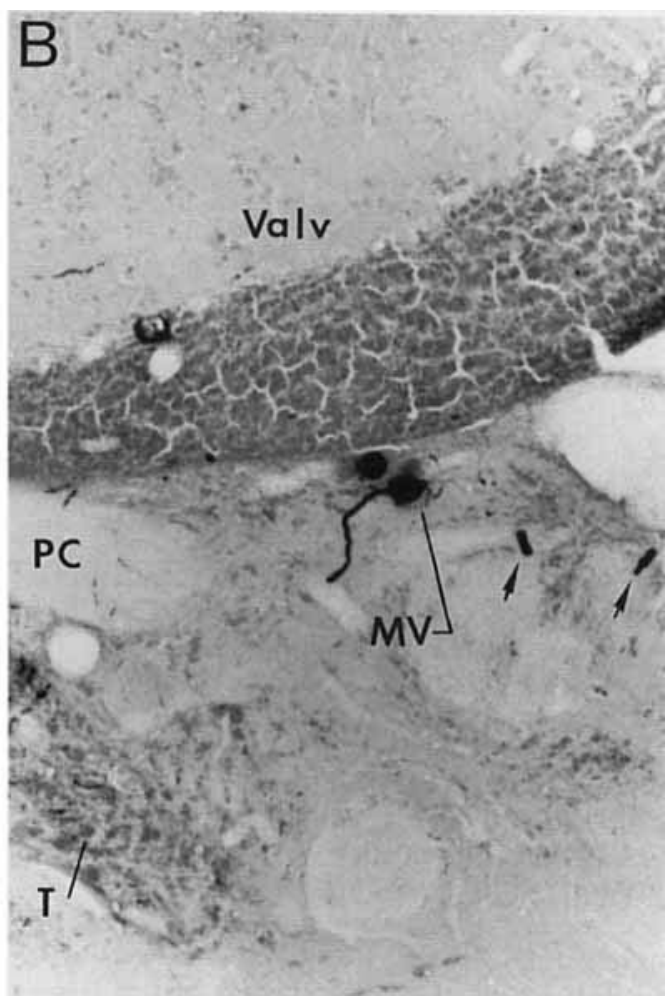

branch. B: Section at the level of posterior commissure showing the labeled soma of two mesencephalic trigeminal neurons 10 days after HRP application to the ophthalmic branch. Arrows indicate two mesencephalic trigeminal fibers whose cell bodies lie more rostrolaterally. Bar scale $=0.15 \mathrm{~mm}$. 


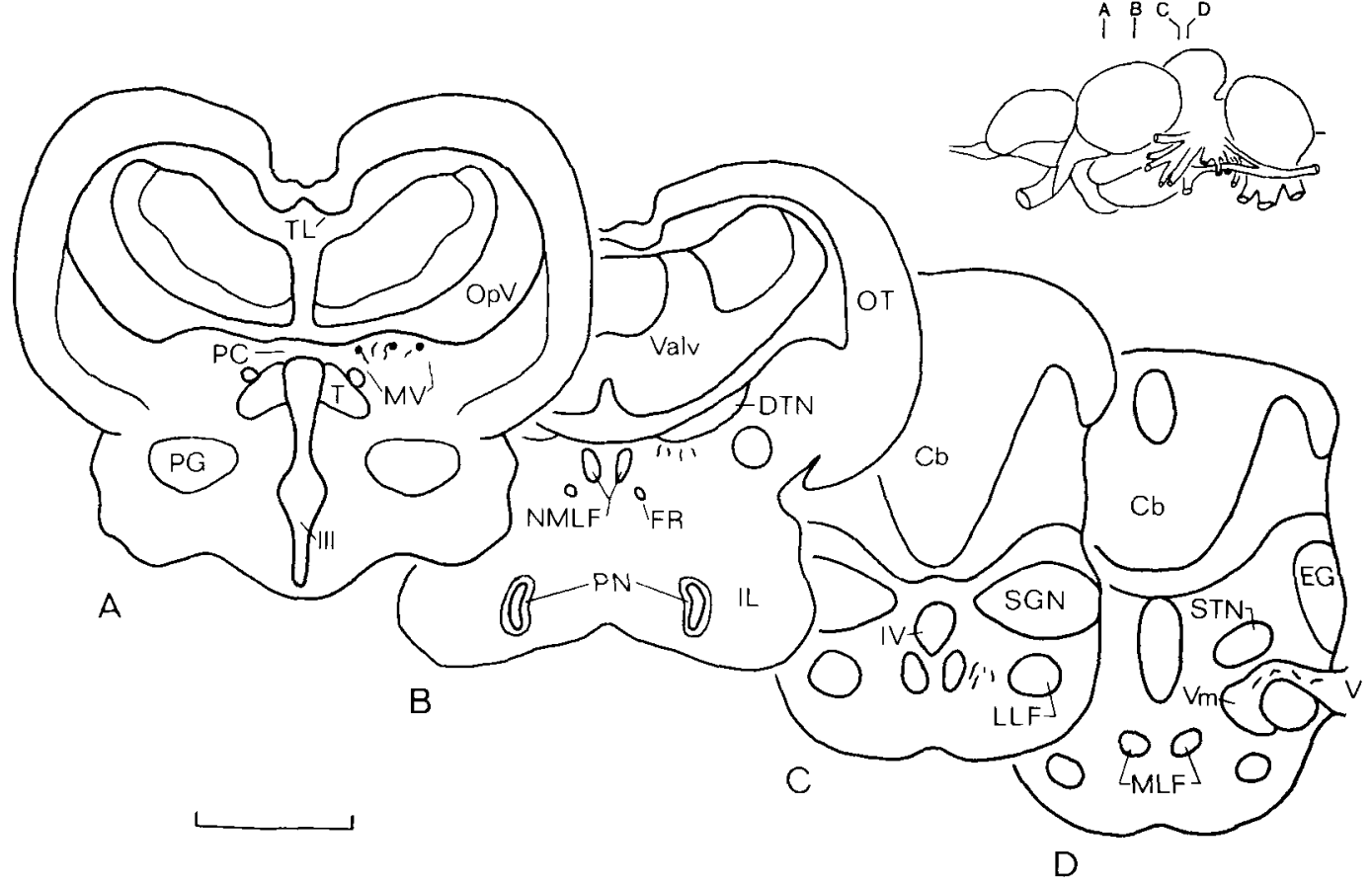

Fig. 8. Drawings of transverse sections through the cerebellum and mesencephalon of Carassius auratus, il lustrating the course of the ascending mesencephalic trigeminal root fibers (short, wavy lines) to their cell bodies (large dots) located along the floor of the ventricle of the optic tectum. Lateral view of the brain in the upper right hand indicates the level of each transverse section. Bar scale $=1 \mathrm{~mm}$. trigeminal and facial motor nuclei. The convergence of tactile and gustatory information upon these cells would provide a quick evaluation of the palatability of a food item and allow rapid ingestion of particularly palatable morsels.

A topographic organization of the primary trigeminal afferents has been reported in a variety of amniotes (Torvik, '56; Kerr, '63; Grant and Arvidsson, '75; Molenaar, '78; Dubbeldam and Karten, '78; Dubbeldam, '80; Barbas-Henry and Lohman, '86). In general the maxillomandibular afferents are described as projecting to the dorsal portion of the trigeminal medullar nuclear complex and the ophthalmic afferents to the ventral portion. In Carassius the organization of the trigeminal afferent projections to the STN and MFn is in accordance with this pattern; the ophthalmic fibers project to the ventral portion of these nuclei and the maxillomandibular afferents project to the dorsal portion.

The cells of the NDTV have been considered to function as interneurons in the proprioceptive reflex circuits of the respiratory muscles (Luiten, '75, '79; Luiten and van der Pers, '77). The projection of the afferents of the ophthalmic branch to the NDTV has important implications in this regard. Unlike the infraorbital and hyomandibular trunks, the supraorbital trunk (of which the ophthalmic branch is a part) innervates no respiratory musculature. The projection of the afferent fibers of the ophthalmic branch to the NDTV suggests that at least a part of this nucleus serves some sensory function (i.e., touch, temperature, pain) other than muscle proprioception.

The projections from the DTV to the trigeminal and facial motor nuclei following labeling of the infraorbital trunk may represent a monosynaptic proprioceptive circuit. Such a circuit was proposed by Luiten ('79) based on electrophysiological recordings from the trigeminal and facial motor nuclei in the carp.

Contralateral trigeminal projections at cervical levels have been described in a variety of tetrapods (Kerr, '63; Fuller and Ebbesson, '73; Molenaar, '78; Dubbeldam and Karten, 
'78; Marfurt, '81; Barbas-Henry and Lohman, '86). In Carassius projections to the contralateral MFn were observed with HRP application to the ophthalmic and infraorbital branches of the trigeminal nerve. Contralateral projections were not observed in previous experimental investigations of the trigeminal afferents in other species of teleosts (Luiten, 75; von Bartheld and Meyer, '85). However, contralateral trigeminal pro jections at cervical levels are present in Ictalurus punctatus and Lepomis cyanellus (personal observations). Therefore, contralateral projections at cervical levels appear to be more widespread among teleosts than previous accounts would indicate.

The failure of previous investigators to report contralateral cervical trigeminal projections in teleosts is probably due to the sparseness of these projections and to differences in techniques rather than to real species differences. The trigeminal afferents in sturgeons, on the other hand, are entirely ipsilateral (New and Northcutt, '84). Thus, there are two patterns for cervical trigeminal projections among osteichthyans. It is uncertain which of these conditions represents the primitive condition for this group. Based on the lack of contralateral cervical projections in hagfishs (Ronan, '85) and lampreys (Northcutt, '79), it is most parsimonious to conclude that contralateral cervical trigeminal projections were not present in the common ancestor of lampreys and gnathostomes. It is possible that the condition seen in tetrapods and teleosts represents an independently derived condition, the lack of contralateral trigeminal projections at cervical levels being the primitive condition for osteichthyans. On the other hand, it is also possible that the pattern seen in sturgeons represents the loss of contralateral projections at cervical levels, the condition found in teleosts and tetrapods representing the primitive condition for osteichthyans. Deciding between these hypotheses will require an investigation of the primary trigeminal afferents in polypteriforms and in elasmobranchs.

Classically, the mesencephalic trigeminal fibers were thought to convey proprioceptive impulses from the muscles of mastication and to course peripherally exclusively in the mandibular branch of the trigeminal nerve (Ariëns Kappers et al., '36). In Carassius the mesencephalic trigeminal sensory fibers are also present in the ophthalmic and maxillary branches of the trigeminal nerve. This distribution of mesencephalic trigeminal fibers agrees with more recent reports on the distribution of the mesencephalic trigeminal fibers in other vertebrates (monitor lizard, Barbas-Henry and Lohman, '86; cat, Corbin, '40; rat, Matesz, '81; dog, Ryu et al., '83).

While there is ample evidence that the mesencephalic nucleus in mammals is involved in proprioception of the masticatory musculature, teeth, tongue, and possibly also of the extrinsic eye muscles (Corbin and Harrison, '40; Dale Smith and Marcarian, '68; Dale Smith, '69; Alvarado-Mallart et al., '75), the function of this nucleus in nonmammals is less well established. Roberts and Witkovsky ('75) demonstrated that the mesencephalic trigeminal neurons in Sycliorhinus and Mustelus do not respond to movements of the jaw, but rather are responsive to mechanical stimulation of the teeth and perioral skin. Luiten ('79) labeled sensory and motor cells in the brainstem of the carp, $C y$ prinus carpio, by intramuscular injection of HRP into the masticatory and extraocular musculature. None of these injections resulted in the labeling of mesencephalic trigeminal cells. Following experiments involving intranerve HRP injection into the maxillary and mandibular branches of the infraorbital trunk, which resulted in the labeling of the mesencephalic trigeminal neurons, Luiten ('79) concluded that the mesencephalic trigeminal neurons serve a perioral mechanoreceptive function. The presence of mesencephalic trigeminal neurons in the supraorbital trunk, which in $\mathrm{Car}$ assius distributes fibers to the rostrodorsal head region, shows that the mesencephalic trigeminal nucleus has a broader peripheral field of innervation than just the perioral region.

The course of the ascending mesencephalic trigeminal fibers in Carassius is similar to what has been described in Salmo and Salvelinus (Woodburne, '36). Projections from the mesencephalic trigeminal neurons to the rostral spinal cord have been described in lepidosirenid lungfishes (Ronan and Northcutt, '85), toads (Corvaja and d'Ascanio, '81), turtles (ten Donkelaar et al., '80), lizards (Goldstein and Jacobs, '69; Ebbesson, '81), snakes (Dacey, '82), and rats (Matesz, '81). In Carassius, Harold Sloan (personal communication) has labeled the mesencephalic trigeminal neurons with HRP application to the rostral spinal cord. Large-diameter fibers, similar to 
the ascending fibers of the mesencephalic trigeminal root, were found in Carassius passing caudally from the level of the entrance of the trigeminal nerve to terminate in the ventromedial MFn. Herrick ('06) considered the MFn to be a coordination center for facial gustatory sensation, mediated via the descending secondary gustatory tract, and for touch sensation, mediated via the descending trigeminal tract. If mesencephalic trigeminal neurons are involved in mechanoreceptive sensation from the rostral head and lips, then it is reasonable that they should project to the coordination center of taste and touch for the head region.

\section{ACKNOWLEDGMENTS}

I thank Dr. R. Glenn Northcutt for his guidance in every aspect of the research for and preparation of this paper. I also thank Dr. M.F. Wullimann, Dr. N.E. Kemp, Dr. B. Oakley, Thomas Schilling, Georg Striedter, and Jiakun Song for critically reading the manuscript, and Martha Vogel for technical assistance in the preparation and sectioning of Paraplast-embedded brains used in this study. This report represents a portion of the author's doctoral dissertation in the Horace H. Rackham School of Graduate Studies at The University of Michigan. This research was supported in part by NIH grants NS1100N and EY02485 to Dr. R.G. Northcutt.

\section{LITERATURE CITED}

Allis, E.P. (1903) The skull and the cranial and first spinal muscles and nerves in Scomber scomber. J. Morphol. 18:45-328.

Alvarado-Mallart, M.R., C. Batini, C. Buisseret-Delmas, and J. Corvisier (1975) Trigeminal representations of the masticatory and extraocular proprioceptors as revealed by horseradish peroxidase retrograde transport Exp. Brain Res. 23:167-179.

Ariëns Kappers, C.U., G.C. Huber, and E.C. Crosby (1936) The Comparative Anatomy of the Nervous Sys. tem of Vertebrates, Including Man. Vol. I. New York: Macmillan. Reprinted 1960: New York: Hafner.

Barbas-Henry, H.A., and A.H.M. Lohman (1988) The motor complex and primary projections of the trigeminal nerve in the monitor lizard, Varanus exanthemati cus. J. Comp. Neurol. 254:314-329.

Corbin, K.B. (1940) Observations on the peripheral distribution of fibers arising in the mesencephalic nucleus of the fifth cranial nerve. J. Comp. Neurol. 73:153-177.

Corbin, K.B., and F. Harrison (1940) Function of mesen cephalic root of fifth cranial nerve. J. Neurophysiol. $3: 423-435$.

Corvaja, N., and P. d'Ascanio (1981) Spinal projections from the mesencephalon in the toad. Brain Behav. Evol. 19:205-213.

Dacey, D.M. (1982) Axon morphology of mesencephalic trigeminal neurons in a snake, Thamnophis sirtalis. J.
Comp. Neurol 204:268-279.

Dale Smith, R. (1969) Location of the neurons innervating tendon spindles of masticator muscles. Exp. Neurol. $25: 646-654$.

Dale Smith, R., and H.Q. Marcarian (1968) Centripetal localization of tooth and tongue tension receptors. J. Dent. Res. 47:616-621.

Dubbeldam, J.L. (1980) Studies on the somatotopy of the trigeminal system in the mallard, A nas platyrhynchos. II. Morphology of the principal sensory nucleus. J. Comp. Neurol. 191:557-571.

Dubbeldam, J.L., and H.J. Karten (1978) The trigeminal system in the pigeon (Columba livia). I. Projections of the gasserian ganglion. J. Comp. Neurol. 180:661-678. Ebbesson, S.O.E. (1981) Projections of the optic tectum and the mesencephalic nucleus of the trigeminal nerve in the tegu lizard (Tupinambis nigropunctatus). Cell Tissue Res. 216:151-165.

Freihofer, W.C. (1966) The Sihler technique of staining nerves for systematic study especially of fishes. Copeia $4: 470-475$.

Fuller, P.M., and S.O.E. Ebbesson (1973) Central projections of the trigeminal nerve in the bull frog (Rano catesbeiana). J. Comp. Neurol. 152:193-200.

Goldstein, M.H., and V.L. Jacobs (1969) The central projections of the mesencephalic root of the trigeminus in a lizard (Lacerta viridis). Brain Res. 14:307-320.

Grant, G., and J. Arvidsson (1975) Transganglionic degeneration in trigeminal primary sensory neurons. Brain Res. 95:285-279.

Hanker, J.S., P.E. Yates, C.B. Metz, and A. Rustioni (1977) A new specific, sensitive and non-carcinogenic reagent for the demonstration of horseradish peroxidase. J. Histochem. 9:789-792.

Harrison, G. (1981) The cranial nerves of the teleost Trichiurus lepturus. J. Morphol. 167:119-134.

Herrick, C.J. (1899) The cranial and first spinal nerves of Menidia; a contribution upon the nerve components of the bony fishes. J. Comp. Neurol. 9:153-455.

Herrick, C.J. (1900) A contribution upon the cranial nerves of the codfish. J. Comp. Neurol. 10:265-318.

Herrick, C.J. (1901) The cranial nerves and cutaneous sense organs of the North American siluroid fishes. J. Comp. Neurol. 11:177-249.

Herrick, C.J. (1906) On the centers for taste and touch in the medulla oblongata of fishes. J. Comp. Neurol. 16:403-439.

Kerr, F. (1983) The divisional organization of the afferent fibers of the trigeminal nerve. Brain 86:721-732.

Luiten, P.G.M. (1975) The central projections of the trigeminal, facial and anterior lateral line nerves in the carp (Cyprinus carpio L.). J. Comp. Neurol. 160:399_ 418.

Luiten, P.G.M. (1979) Proprioceptive reflex connections of head musculature and the mesencephalic trigeminal nucleus in the carp. J. Comp. Neurol. 183:903-912.

Luiten, P.G.M., and J.N.C. van der Pers (1977) The connections of the trigeminal and facial motor nuclei in the brain of the carp (Cyprinus carpio L.) as revealed by anterograde and retrograde transport of horseradish peroxidase. J. Comp. Neurol. 174:575-590.

Maheshwari, S.C. (1965) The cranial nerves of Mastacembelus armatus. Jpn. J. Ichthyol. 12:89-98.

Marfurt, C.F. (1981) The central projections of trigeminal primary afferent neurons in the cat as determined by the transganglionic transport of horseradish peroxidase. J. Comp. Neurol. 203:785-798.

Matesz, C. (1981) Peripheral and central distribution of fibers of the mesencephalic trigeminal root in the rat. Neurosci. Lett. 27:13-17.

Mesulam, M.M. (1978) Tetramethyl benzidine for horse radish peroxidase neurohistochemistry. A non-carci- 
nogenic blue reaction-product with superior sensitivity for visualizing neural afferents and efferents. J. Histochem. Cytochem. 28:108-117.

Molenaar, G.J. (1978) The sensory trigeminal system of a snake in the possession of infrared receptors. II. The central projections of the trigeminal nerve. J. Comp. Neurol. 179:137-152.

New, J.G., and R.G. Northcutt (1984) Primary projections of the trigeminal nerve in two species of sturgeon: Acipenser oxyrhynchus and Scaphirhynchus platyrhynchus. J. Morphol. 182:125-136.

Nieuwenhuys, R., and E. Pouwels (1983) The brain stem of actinopterygian fishes. In R.G. Northeutt and R.E. Davis (eds): Fish Neurobiology. Vol. 1. Ann Arbor, Michigan: University of Michigan Press, pp. 25-87.

Northeutt, R.G. (1978) Elasmobranch brain organization. In S. Hodgson and R.F. Mathewson (eds): Sensory Biology of Sharks, Skates and Rays. Arlington, Virginia: U.S. Government Printing Office, pp. 117-193.

Northcutt, R.G. (1979) Experimental determination of the primary trigeminal projections in lampreys. Brain Res, 163:323-327.

Olszewski, J. (1950) On the anatomical and functional organization of the spinal trigeminal nucleus. J. Comp. Neurol. 92:401-413.

Puzdrowski, R.L. (1985) The primary projections of the trigeminal nerve in goldfish, Carassius auratus. Soc. Neurosci. Abstr. 11:1311.

Puzdrowski, R.L. (1987) The peripheral distribution and central projections of the sensory rami of the facial nerve in goldfish, Carassius auratus. J. Comp. Neurol. 259:382-392.

Roberts, R.L., and P. Witkovsky (1975) A functional analysis of the mesencephalic nucleus of the fifth nerve in the selachian brain. Proc. R. Soc. Lond. B, 190:473495.

Ronan, M. (1985) Primary trigeminal projections in the
Pacific hagfish. Soc. Neurosci. Abstr. 11:1311.

Ronan, M., and R.G. Northcutt (1985) The origins of descending spinal projections in lepidosirenid lung. fishes. J. Comp. Neurol. 241:435-444.

Ryu, K., K. Watanabe, and E. Kawana (1983) The mesencephalic root fibers of the trigeminal nerve in the dog. Acta Anat. 116:28-36.

Saxena, P.K. (1967) The cranial nerves of the mud eel Amphipnous cuchia. Acta Anat. 87:306-320.

Smeets, W.J.A.J., and R. Nieuwenhuys (1978) Topological analysis of the brain stem of the sharks Squalus acanthias and Scyliorhinus canicula. J. Comp. Neurol. 106:51-142.

ten Donkelaar, H.J., A. Kusuma, and R. de Boer-van Huizen (1980) Cells of origin of pathways descending to the spinal cord in some quadrupedal reptiles. J. Comp. Neurol. 192:827-851.

Torvik, A. (1956) Afferent connections to the sensory trigeminal nuclei, the nucleus of the solitary tract and adjacent structures. An experimental study in the rat. J. Comp. Neurol. 106:51-142.

von Bartheld, C.S., and D.L. Meyer (1985) Trigeminal and facial innervation of cirri in three teleost species. Cell Tissue Res. 241:615-622.

Weinberg, E. (1968) The mesencephalic root of the fifth nerve. A comparative anatomical study. J. Comp. Neurol. 48:249-405.

Woodburne, R.T. (1936) Phylogenetic considerations of the primary and secondary centers and connections of the trigeminal complex in a series of vertebrates. J. Comp. Neurol. 65:403-493.

Wullimann, M.F., and R.G. Northcutt (1986) Afferent connections of the valvula cerebelli in the goldfish, Carassius auratus. In Splechtna and Hilgers (eds): 2nd Int. Symp. Vert. Morphol. Vienna, Fortsehr. Zool. No. 35. Stuttgart; Fischer (in press). 